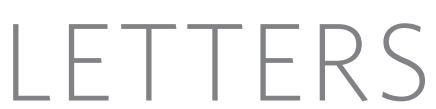

\title{
Regulation of parkinsonian motor behaviours by optogenetic control of basal ganglia circuitry
}

\author{
Alexxai V. Kravitz ${ }^{1}$, Benjamin S. Freeze ${ }^{1,4,5}$, Philip R. L. Parker ${ }^{1,3}$, Kenneth Kay ${ }^{1,5}$, Myo T. Thwin ${ }^{1}$, Karl Deisseroth ${ }^{6}$ \\ \& Anatol C. Kreitzer ${ }^{1,2,3,4,5}$
}

Neural circuits of the basal ganglia are critical for motor planning and action selection ${ }^{1-3}$. Two parallel basal ganglia pathways have been described ${ }^{4}$, and have been proposed to exert opposing influences on motor function ${ }^{5-7}$. According to this classical model, activation of the 'direct' pathway facilitates movement and activation of the 'indirect' pathway inhibits movement. However, more recent anatomical and functional evidence has called into question the validity of this hypothesis ${ }^{8-10}$. Because this model has never been empirically tested, the specific function of these circuits in behaving animals remains unknown. Here we report direct activation of basal ganglia circuitry in vivo, using optogenetic control ${ }^{11-14}$ of direct- and indirect-pathway medium spiny projection neurons (MSNs), achieved through Cre-dependent viral expression of channelrhodopsin-2 in the striatum of bacterial artificial chromosome transgenic mice expressing Cre recombinase under control of regulatory elements for the dopamine D1 or D2 receptor. Bilateral excitation of indirect-pathway MSNs elicited a parkinsonian state, distinguished by increased freezing, bradykinesia and decreased locomotor initiations. In contrast, activation of directpathway MSNs reduced freezing and increased locomotion. In a mouse model of Parkinson's disease, direct-pathway activation completely rescued deficits in freezing, bradykinesia and locomotor initiation. Taken together, our findings establish a critical role for basal ganglia circuitry in the bidirectional regulation of motor behaviour and indicate that modulation of direct-pathway circuitry may represent an effective therapeutic strategy for ameliorating parkinsonian motor deficits.

To obtain selective optogenetic control of the direct and indirect pathways in vivo, we targeted striatal MSNs that form the origin of these pathways. We injected an adeno-associated virus (AAV1) containing a double-floxed inverted open reading frame encoding a fusion of channelrhodopsin-2 and enhanced yellow fluorescent protein ${ }^{15,16}$ (ChR2-YFP) (Fig. 1a) into the dorsomedial striatum of bacterial artificial chromosome (BAC) transgenic mice expressing Cre recombinase in direct- or indirect-pathway $\mathrm{MSNs}^{17}$ (D1-Cre and D2-Cre mice, respectively). Functional ChR2-YFP is transcribed only in neurons containing Cre, thus restricting expression to either direct- or indirect-pathway MSNs. Dorsomedial striatum was chosen as a target because it is thought to be involved in earlier stages of motor processing $^{1}$ and thus would be more likely to yield global changes in motor behaviour.

To confirm the expression pattern of ChR2 in the dorsomedial striatum, we prepared sagittal sections at two weeks post-injection that included the striatum, globus pallidus and substantia nigra pars reticulata (SNr) (Fig. 1b). In D1-Cre mice, numerous ChR2-YFPpositive cell bodies were observed in the striatum, along with fibres traversing the globus pallidus that projected to the entopeduncular nucleus and $\mathrm{SNr}$ (Fig. 1c), which are the canonical targets of directpathway MSNs. In D2-Cre mice, ChR2-YFP-positive cell bodies were
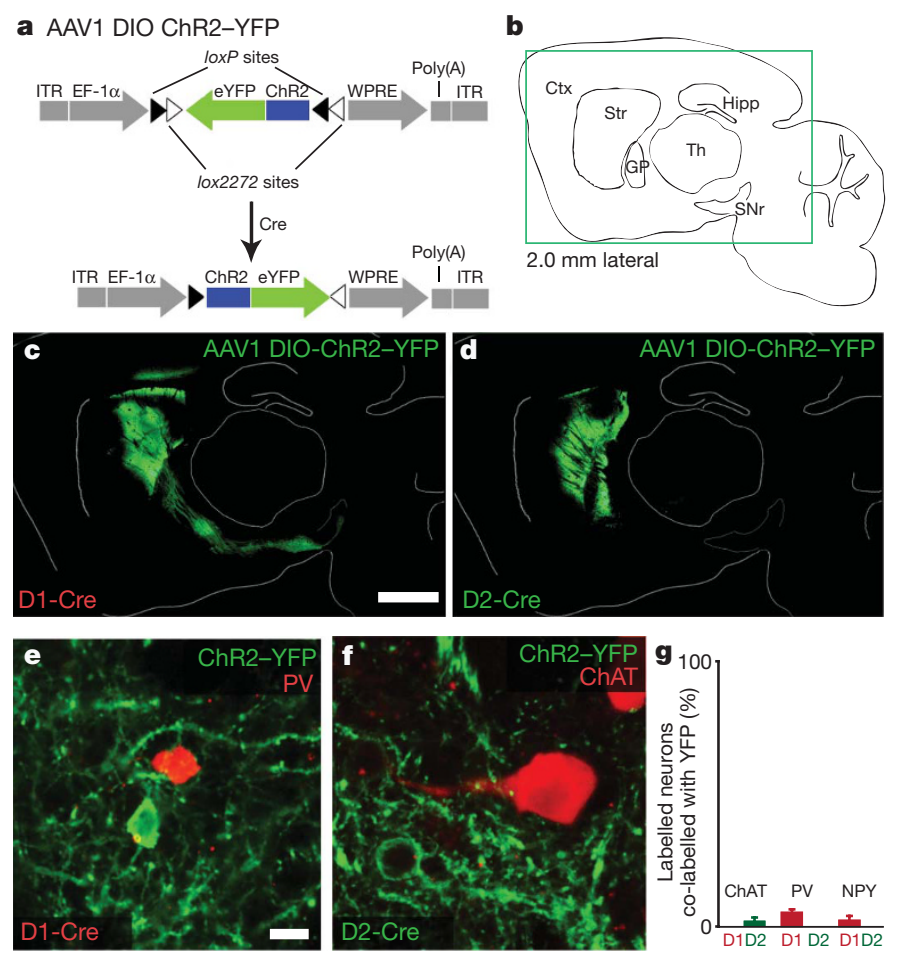

Figure 1 | Selective viral-mediated ChR2 expression in striatal direct- or indirect-pathway MSNs. a, Schematic of the double-floxed Cre-dependent AAV vector expressing ChR2-YFP under control of the EF- $1 \alpha$ promoter. DIO, double-floxed inverted open reading frame; eYFP, enhanced YFP; ITR, inverted terminal repeat; WPRE, woodchuck hepatitis virus post-

transcriptional regulatory element. b, Sagittal mouse brain schematic. Ctx, cortex; Str, striatum; GP, globus pallidus; SNr, substantia nigra pars reticulata; Th, thalamus; Hipp, hippocampus. The box indicates the region shown in panels c and d. c, Sagittal section showing striatal direct-pathway MSNs expressing ChR2-YFP following injection of Cre-dependent AAV1 into D1-Cre BAC transgenic mice. Direct-pathway MSN axons target the SNr. d, Expression of ChR2-YFP in striatal indirect-pathway MSNs of D2Cre BAC transgenic mice. Indirect-pathway MSN axons target the GP. Scale bars in $\mathbf{c}$ and $\mathbf{d}, 1 \mathrm{~mm}$. e, $\mathbf{f}$, Examples of ChR2-YFP-expressing neurons that do not co-express the interneuronal markers parvalbumin (PV) and choline acetyltransferase (ChAT). Scale bars in e and f, $15 \mu \mathrm{m}$. g, Percent of ChAT, PV or neuropeptide Y neurons that co-express ChR2-YFP. Error bars, s.e.m. 
observed in the striatum, and fibres projected to the globus pallidus but not the entopeduncular nucleus or $\mathrm{SNr}$, consistent with proper targeting of ChR2-YFP to indirect-pathway MSNs (Fig. 1d). Immunostaining for Cre and the MSN marker DARPP-32 ${ }^{18}$ (also known as PPP1R1B) confirmed that the vast majority (>90\%) of ChR2-YFP-positive cells expressed both Cre and DARPP-32 (Supplementary Fig. 1 and Supplementary Table 1).

To investigate whether ChR2-YFP was expressed in striatal interneurons, we immunostained each line for choline acetyltransferase, parvalbumin and neuropeptide Y (Fig. 1e-g), which are markers of cholinergic, fast-spiking and low-threshold-spiking interneurons, respectively ${ }^{19}$. Very few $(<5 \%)$ interneurons expressed ChR2-YFP (Fig. 1g and Supplementary Table 1). These findings were confirmed electrophysiologically, by comparing the passive and active membrane properties of ChR2-YFP-positive neurons with the properties of known striatal neuron subtypes ${ }^{20}$. All recorded neurons exhibited characteristics of MSNs (Supplementary Fig. 2 and Supplementary Table 1). We found no evidence for expression of ChR2-YFP in striatal afferent fibres from either the substantia nigra pars compacta (Supplementary Fig. 3) or cortex (Supplementary Fig. 4).

To confirm that ChR2-YFP expression alone did not alter the electrophysiological properties of MSNs, we performed whole-cell recordings in brain slices prepared from D1-Cre or D2-Cre mice injected with ChR2-YFP virus (subsequently referred to as D1ChR2 or D2-ChR2 mice, respectively). The current-firing relationships for direct- and indirect-pathway MSNs expressing ChR2-YFP were not significantly different from control MSNs (D1-ChR2 versus control: $P>0.30$ for all points; D2-ChR2 versus control: $P>0.26$ for all points; Fig. 2a, b), and ChR2-YFP expression did not significantly change the passive properties of MSNs (Supplementary Table 2). However, consistent with previous reports ${ }^{21}$, D1-ChR2-positive MSNs were significantly less excitable than D2-ChR2-positive MSNs, providing further evidence that these subpopulations were selectively labelled by ChR2-YFP. Illumination of ChR2-YFPpositive MSNs with 470-nm light elicited large light-evoked currents in voltage clamp and robust spiking in current clamp (Fig. 2c, d), indicating that ChR2 was functional in these neurons.

We next tested ChR2 function in vivo in the striatum of anaesthetized D1-ChR2 and D2-ChR2 mice. Recordings were performed with an optrode $^{22}$ that consisted of a linear, 16-site silicon probe with an integrated laser-coupled optical fibre that could elicit light-induced spiking at least $800 \mu \mathrm{m}$ from the fibre tip (Supplementary Fig. 5). In both mouse lines, we observed significant firing rate increases in approximately $35 \%$ of recorded neurons during $473-\mathrm{nm}$ laser illumination ( $1 \mathrm{~mW}$ at fibre tip) (Fig. 2e-h), although this is probably an overestimate of the actual percentage of ChR2-positive MSNs. We considered the possibility that illumination recruited previously silent neurons that could infiltrate the recording and bias our quantification of firing rate changes. However, we observed no difference in the spike waveforms during illumination (Fig. 2f, g, insets), nor did lightinduced spikes occur within the refractory period of the recorded neuron, indicating that no additional units were recruited. Overall, average MSN firing rates in D1-ChR2 mice increased from $0.03 \mathrm{~Hz}$ to $1.16 \mathrm{~Hz}$ with illumination; in $\mathrm{D} 2-\mathrm{ChR} 2$ mice, average firing rates

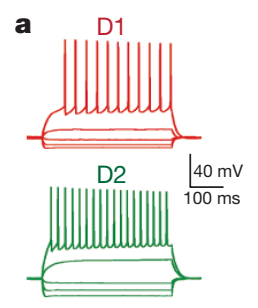

b
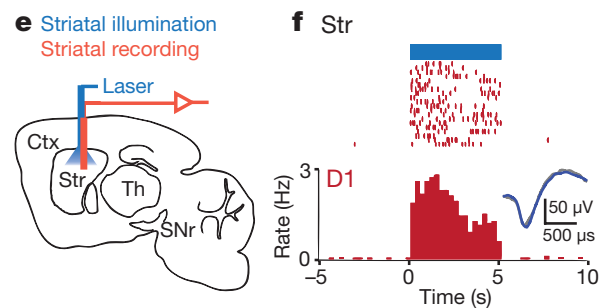

i Striatal illumination
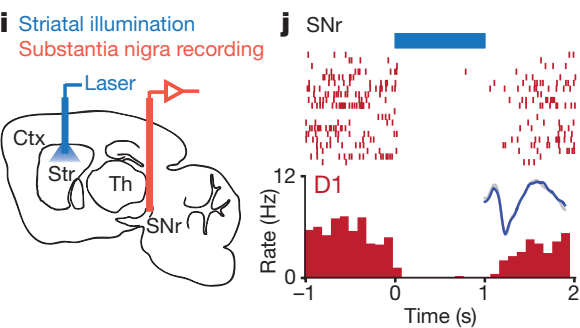

Figure 2 | ChR2-mediated excitation of direct- and indirect-pathway MSNs in vivo drives activity in basal ganglia circuitry. a, Whole-cell current-clamp recordings from ChR2-YFP-positive neurons in vitro demonstrate normal current-firing relationships consistent with direct-pathway (red traces) and indirect-pathway (green traces) MSNs (D1-control, $n=10$; D1-ChR2, $n=3$; D2-control, $n=7$; D2-ChR2, $n=3$ ). b, Firing rate plotted as a function of injected current in direct- and indirect-pathway MSNs expressing either green fluorescent protein or ChR2-YFP. c, ChR2-mediated photocurrents (top) and spiking (bottom) in direct-pathway (left) and indirect-pathway (right) MSNs. In this and subsequent panels, blue bars indicate illumination time. d, Summary of ChR2-mediated photocurrents (left) and spiking (right) for D1-ChR2 $(n=5)$ and D2-ChR2 $(n=4)$ cells. e, Schematic of in vivo optical stimulation and recording in the striatum. f, Example MSN c
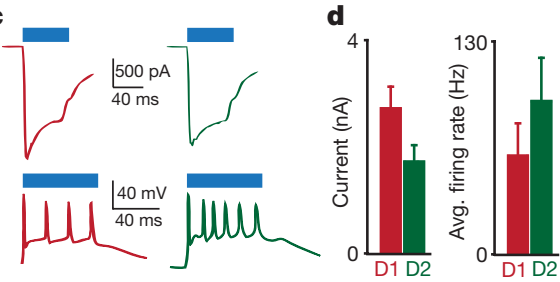

g $\operatorname{Str}$
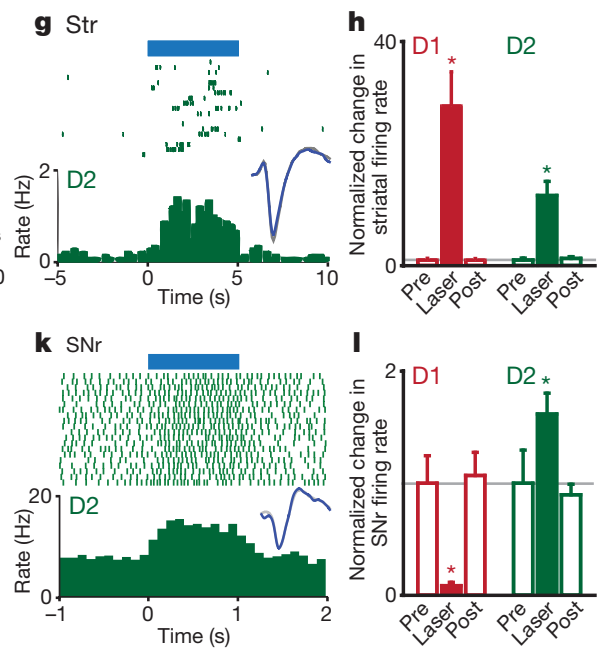

recorded from the striatum of an anaesthetized D1-ChR2 mouse that showed increased firing in response to illumination. Insets in $\mathbf{f}, \mathbf{g}, \mathbf{j}$ and $\mathbf{k}$ show spike waveforms with (blue) or without (grey) illumination. Scale bars apply to insets in $\mathbf{f}, \mathbf{g}, \mathbf{j}$ and $\mathbf{k}$. $\mathbf{g}$, Example of a light-sensitive MSN from a D2-ChR2 mouse. $\mathbf{h}$, Normalized change in MSN firing rates in response to striatal illumination in D1-ChR2 $(n=16)$ and D2-ChR2 $(n=10)$ mice. Pre, before illumination; laser, during illumination; post, after illumination. i, Schematic of in vivo optical stimulation in striatum and recording in $\mathrm{SNr}$. j, Example of a SNr neuron recorded from a D1-ChR2 mouse that was inhibited by direct-pathway activation. $\mathbf{k}$, Example of a $\mathrm{SNr}$ neuron recorded from a D2-ChR2 mouse that was excited by indirect-pathway activation.

I, Normalized change in $\mathrm{SNr}$ firing rate in response to activation of the direct $(\mathrm{D} 1, n=8)$ or indirect $(\mathrm{D} 2, n=4)$ pathways. ${ }^{*} P<0.05$; error bars, s.e.m. 
increased from $0.06 \mathrm{~Hz}$ to $0.76 \mathrm{~Hz}$ with illumination (D1-ChR2: $n=16, P<0.0001$; D2-ChR2: $n=10, P<0.005)$. The light-induced firing rate of MSNs $(\sim 1 \mathrm{~Hz})$ was well below the maximal firing rate of MSNs, indicating that we did not drive these neurons strongly under conditions of anaesthesia. However, basal MSN firing rates under anaesthesia were approximately tenfold lower than those observed in awake mice, suggesting that our light-induced firing rate changes may not reflect the efficacy of optical stimulation in awake mice.

According to the classical model of basal ganglia function, selective expression of dopamine D1 receptor and dopamine D2 receptor in the direct and indirect pathways, respectively, allows differential modulation of direct- and indirect-pathway $\mathrm{MSNs}^{5,23}$. To test this hypothesis experimentally, we recorded from optically identified direct- or indirect-pathway MSNs in vivo and administered D1 or D2 agonists. However, no consistent effects were observed (Supplementary Fig. 6), highlighting the complexity of pharmacological modulation in intact circuits.

To confirm that activation of direct- or indirect-pathway MSNs can drive activity in basal ganglia circuits in vivo, we next performed recordings in the main basal ganglia output nucleus $(\mathrm{SNr})$ during striatal illumination in D1-ChR2 or D2-ChR2 mice (Fig. 2i). In SNr regions innervated by optically stimulated MSNs (as assessed by local field potential modulation), eight of ten $\mathrm{SNr}$ neurons responded to direct-pathway activation, whereas four of eleven $\mathrm{SNr}$ neurons responded to indirect-pathway activation (Supplementary Fig. 7). All responsive $\mathrm{SNr}$ neurons showed robust changes in firing rate (D1-ChR2 mice: $8.6 \pm 3.0 \%$ of baseline firing rate, $n=8$; D2-ChR2 mice: $162 \pm 19 \%$ of baseline firing rate, $n=4$ ) that were consistent with the classical model: direct-pathway activation inhibited firing of $\mathrm{SNr}$ neurons, whereas indirect-pathway activation excited $\mathrm{SNr}$ neurons (Fig. 2j-1).

After verifying the expression pattern of ChR2 in the direct or indirect pathways, and confirming our ability to drive direct- and indirect-pathway basal ganglia circuits in vivo, we examined the behavioural effects of activating basal ganglia circuits in awake mice. Cannulae were surgically implanted over dorsomedial striatum (Fig. 3a) and used to guide both viral injections and fibre-optic placements. Unilateral illumination of dorsomedial striatum in D1-ChR2 and D2-ChR2 mice elicited rotational behaviour (Supplementary Fig. 8; also see Supplementary Movie 1). Directpathway activation led to contraversive rotations, whereas indirectpathway activation yielded ipsiversive rotations. Thus, unilateral indirect-pathway activation mimics rotational behaviour induced by unilateral dopamine depletion ${ }^{24}$, consistent with the classical model of basal ganglia function.

Bilateral illumination of direct-pathway MSNs elicited decreases in freezing and fine movements and an increase in the percentage of time spent in ambulation ( $n=9, P<0.05$; Fig. $3 \mathrm{~b}, \mathrm{c})$. In contrast, when we stimulated the indirect pathway, we observed decreases in ambulation and fine movements $(n=8, P<0.05)$ and a sharp increase in the amount of time spent freezing $(n=8, P<0.001$; Fig. 3b, c; also see Supplementary Movie 2). Changes in the percentage of time spent performing fine movements were not correlated with changes in grooming frequency for either ChR2-expressing line (D1-ChR2: $n=9, P=0.30$; D2-ChR2: $n=8, P=0.93)$. However, fine movements were more vigorous during direct-pathway activation (increased centre-point velocity) and less vigorous during indirect-pathway activation (D1-ChR2: $n=9, P>0.005$; D2-ChR2: $n=8, P>0.05$; Fig. 3d). As a control, we injected Cre-dependent virus expressing only YFP (D1-YFP and D2-YFP mice). We observed no difference in time spent ambulatory, freezing or performing fine movements in either of these mouse lines during illumination $(n=5$ for each line, $P>0.23$ for all conditions).

Next we analysed ambulation patterns during bilateral activation of the direct or indirect pathways (Fig. 3e-g). During illumination, D1-ChR2 mice initiated more frequent ambulatory bouts $(n=9$, $P<0.005)$, and these bouts lasted longer $(n=9, P<0.05)$; no
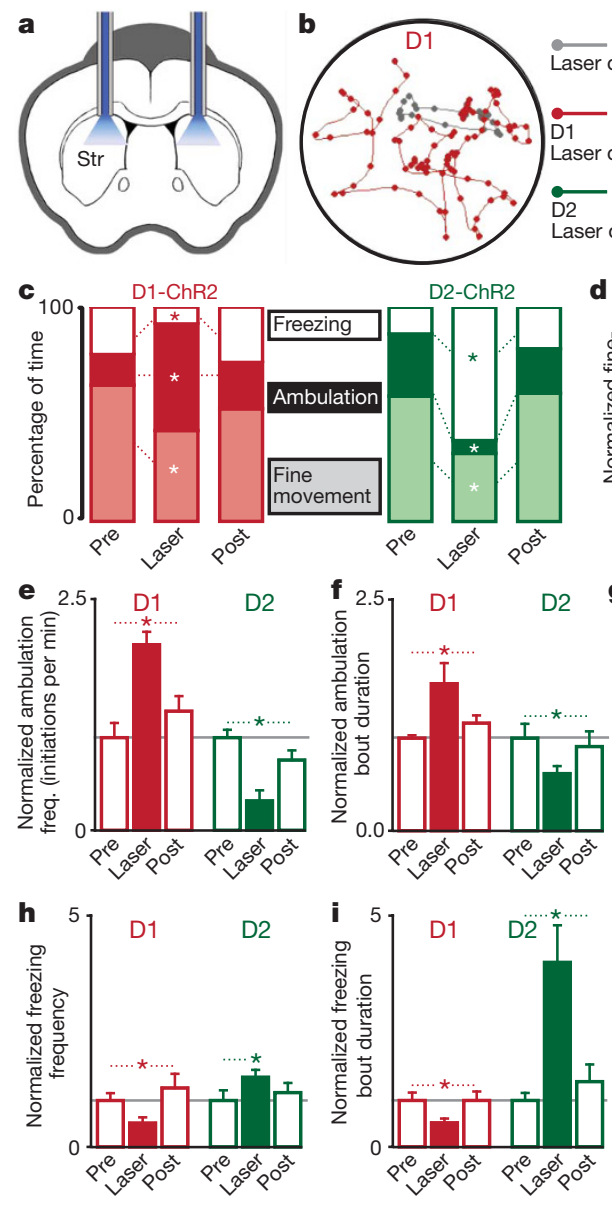
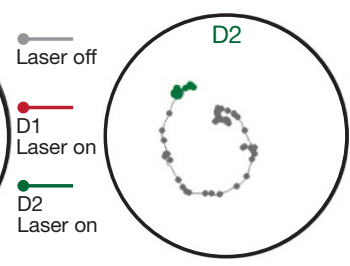

D2-ChR2

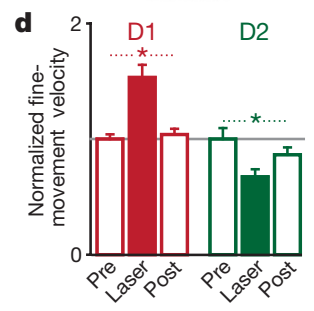

$f_{2.5}$ D1 D2
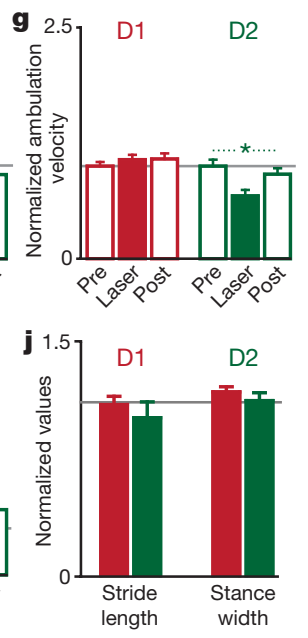

Figure 3 | In vivo activation of direct or indirect pathways reveals pathwayspecific regulation of motor function. a, Coronal schematic of cannula placement and bilateral fibre-optic stimulation. $\mathbf{b}$, Example of altered motor activity during bilateral striatal illumination in D1-ChR2 (left) and D2ChR2 (right) mice. Lines represent the mouse's path; dots represent the mouse's location every $300 \mathrm{~ms}$. Grey paths represent $20 \mathrm{~s}$ of activity before illumination; coloured paths represent $20 \mathrm{~s}$ of activity during subsequent illumination. c, Motor activity before, during and after bilateral striatal illumination in D1-ChR2 (left, red) and D2-ChR2 (right, green) mice. d-i, Effect of illumination on the velocity of fine movements (d), initiation of ambulatory bouts (e), ambulation bout duration (f), ambulation velocity (g), frequency of freezing (h) and duration of freezing bouts (i) in D1-ChR2 (red bars, $n=9$ ) and D2-ChR2 (green bars, $n=8$ ) mice. j, No change in gait in response to illumination in D1-ChR2 (red bars, $n=4$ ) or D2-ChR2 (green bars, $n=5$ ) mice. ${ }^{*} P<0.05$; error bars, s.e.m.

changes in ambulation velocity occurred ( $n=9, P=0.21)$. In contrast, indirect-pathway activation led to less frequent initiation of ambulation $(n=8, P<0.0005)$. When ambulation did occur, it lasted a shorter time $(n=8, P<0.05)$ and had a lower velocity $(n=8, P<0.0005)$. There were no differences in any measures of ambulation for the YFP-expressing controls during illumination ( $n=5$ for each line, $P>0.35$ for all measures; Supplementary Fig. 9). We then examined the frequency and duration of freezing bouts during illumination (Fig. 3h, i). D1-ChR2-expressing mice exhibited less frequent freezing bouts $(n=9, P<0.05)$, and these bouts were shorter $(n=9, P<0.0001)$. D2-ChR2 expressing mice had more frequent freezing bouts during illumination $(n=8, P<0.05)$, and freezing bouts that did occur lasted much longer $(n=8, P<0.05)$. There were no differences in either measure of freezing for the YFPexpressing controls during illumination ( $n=5$ for each line, $P>0.20$ for each measure; Supplementary Fig. 9). Together, these data establish a causal role for the direct pathway in decreasing freezing and increasing locomotor initiations, and for the indirect pathway in increasing freezing, decreasing locomotor initiations and inducing bradykinesia. 
To test whether changes in ambulation patterns reflected altered locomotor coordination, we examined gait parameters in D1-ChR2 and D2-ChR2 mice in response to illumination, using a treadmill equipped with a high-speed camera. We quantified multiple gait parameters with the laser on and off, and found no significant differences in the average or variance of stride length, stance width, stride frequency, stance duration, swing duration, paw angle and paw area on belt for either line ( $n=4$ for D1-ChR2, $n=5$ for D2-ChR2, $P>0.05$ for all; Fig. 3j and Supplementary Fig. 10). This indicates that activation of direct and indirect pathways in the dorsomedial striatum regulates the pattern of motor activity, without changing the coordination of ambulation itself.

Parkinsonian motor deficits are proposed to result from an overactive indirect pathway and an underactive direct pathway ${ }^{5,25-27}$. Surgical treatments for Parkinson's disease are focused on reducing activity in the indirect pathway, through lesions or deep-brain
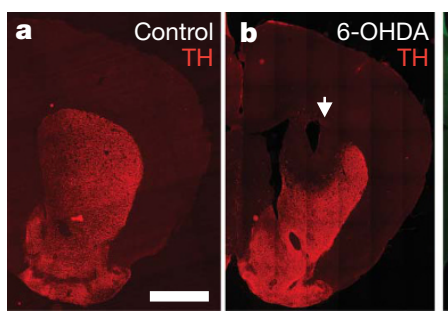

e Pre-lesion D1-ChR2 + 6-OHDA
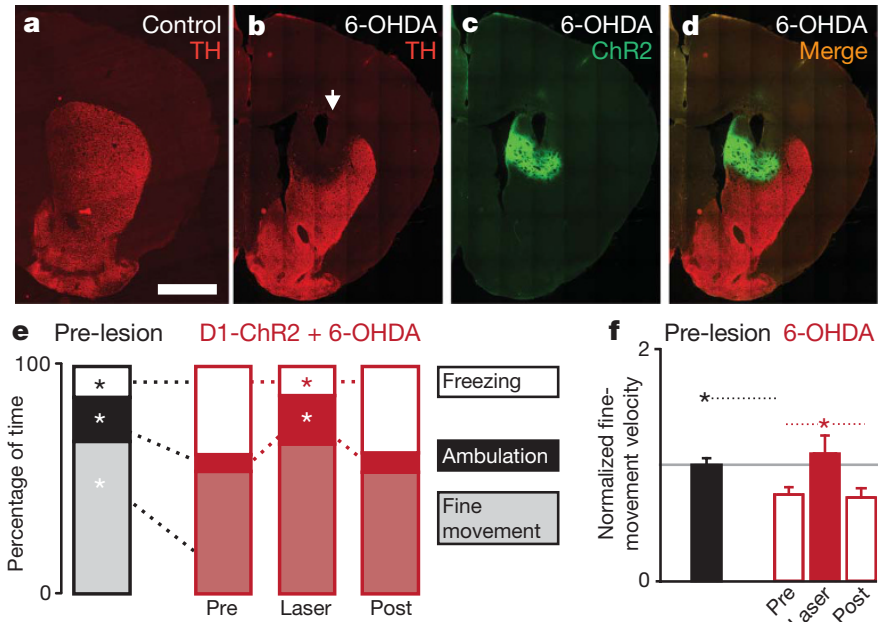

f Pre-lesion 6-OHDA
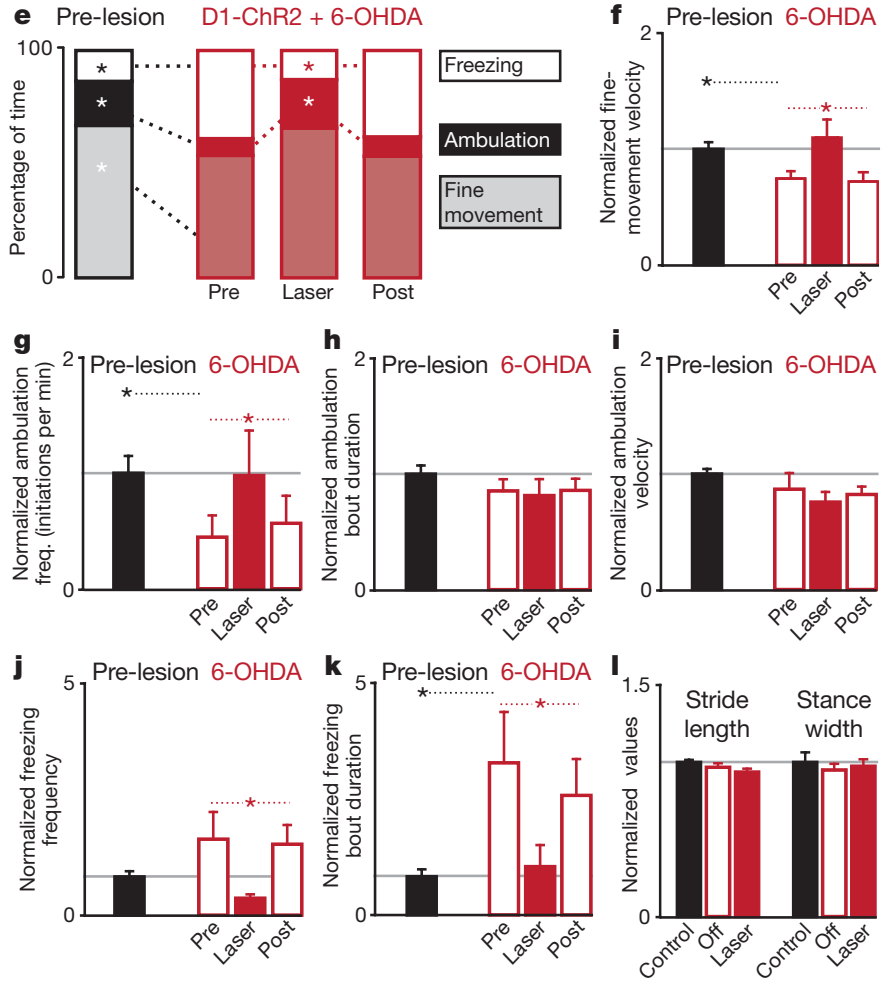

Figure 4 | Direct-pathway activation rescues motor deficits in the 6-OHDA model of Parkinson's disease. a, Visualization of striatal dopaminergic afferents by tyrosine hydroxylase (TH) staining in coronal slices. Scale bar, $1 \mathrm{~mm}$. b. Loss of dopaminergic innervation in dorsomedial striatum one week after 6-OHDA injection. The arrow marks the injection site. c, ChR2-YFP expression in dorsomedial striatum of 6-OHDA-lesioned mice. d, Merged image shows overlap of ChR2 expression with the 6-OHDA lesion. e, Motor behaviour before (left, black bars) and after (right, red bars) 6-OHDA lesion in D1-ChR2 mice $(n=10)$. In 6-OHDA-lesioned mice, behaviour is shown before, during and after activation of the direct pathway. $\mathbf{f}-\mathbf{k}$, Effect of 6-OHDA lesion and direct pathway rescue on fine-movement velocity (f), initiation of ambulatory bouts $(\mathbf{g})$, ambulation bout duration (h), ambulation velocity (i), frequency of freezing (j) and duration of freezing bouts (k). I, No change in gait was observed after 6-OHDA lesioning or direct-pathway activation. ${ }^{*} P<0.05$; error bars, s.e.m. stimulation. However, there has been no experimental test of whether increasing direct-pathway activity in Parkinson's disease models can ameliorate parkinsonian motor symptoms. We tested this hypothesis using bilateral illumination of ChR2 in bilateral 6-hydroxydopamine (6-OHDA)-lesioned mice. We injected 6-OHDA bilaterally into the dorsomedial striatum, resulting in a near-total loss of dopaminergic innervation of this region after one week (tyrosine hydroxylase intensity in dorsomedial striatum: $4 \pm 12 \%$ of control, $n=4, P<0.005$; Fig. 4 a, b), which was accompanied by parkinsonian motor deficits that included bradykinesia (decreased fine-movement velocity), decreased time spent ambulatory, decreased locomotor initiation and increased freezing (both frequency and duration) $(n=10, P<0.05$ for all analyses; Fig. 4e-k). We attempted to rescue these symptoms using bilateral direct-pathway activation in the same region of dorsomedial striatum that lacked dopamine innervation (Fig. 4c, d; also see Methods). Unexpectedly, direct-pathway activation completely restored motor behaviour to pre-lesion levels (Fig. 4e-k). Direct-pathway activation eliminated bradykinesia (increased fine-movement velocity to prelesion levels, $P<0.01)$, increased locomotor initiations $(P<0.05)$ and decreased freezing $(P<0.05)$. Changes in gait parameters were not observed after 6-OHDA injection or direct-pathway activation $(P>0.14$; Fig. 4l). Taken together, these findings provide the first evidence that direct-pathway activation can ameliorate a constellation of motor deficits in a mouse model of Parkinson's disease. However, future studies using a comprehensive battery of sensorimotor tests will be required to characterize fully the role of direct- and indirect-pathway circuit activity in motor function and dysfunction in Parkinson's disease.

\section{METHODS SUMMARY}

Viral expression of DIO-ChR2-YFP in BAC transgenic mice. Injections of recombinant AAV1 virus targeted the dorsomedial striatum in BAC transgenic D1-Cre and D2-Cre (BAC) transgenic mice ${ }^{17}$. DIO constructs encoding ChR2YFP restricted expression to Cre-positive cells ${ }^{15,16}$.

Electrophysiology. For in vivo recordings, we coupled an $80-\mathrm{mW}$ through-thelens-controlled 473-nm laser to a silicon optrode (NeuroNexus) using a fibre-optic patch cord, and attenuated the laser power to yield $1 \mathrm{~mW}$ at the fibre tip. Recordings were performed using a 64-channel multichannel acquisition processor (Plexon). Single units were discriminated with principal component analysis.

Optical stimulation and behavioural analysis in awake mice. Two 3-m glass fibres (AFS105/125Y, Thorlabs) were connectorized with SMA connectors at one end and cleaved flat at the other end, and were inserted through guide cannulae into the dorsomedial striatum. The laser power was adjusted to yield $1 \mathrm{~mW}$ at tip of each fibre. The positions of the nose, tail and centre of mass of each mouse were tracked using Noldus ETHOVISION 7.0 software. Animals were recorded for a 1-10-min baseline period before any laser illumination, to obtain baseline behavioural measures. Then the striatum was illuminated in a series of 10 trials. Each trial had a variable period during which the laser was on (constant illumination, 30-s average, 20-40-s range) followed by a variable period in which the laser was off (60-s average, 50-70-s range).

6-OHDA treatment. Mice were cannulated and injected with ChR2-YFP as described above. Nine days after viral injection, pre-lesion behavioural data was collected from these animals. Directly following this data collection, mice were anaesthetized with ketamine and xylazine, and 6-OHDA $\left(5 \mathrm{mg} \mathrm{ml}^{-1}\right)$ was injected through the same cannulae as the virus ( $1 \mu$ l per hemisphere). Mice were allowed to recover for five days before post-lesion behavioural testing.

Full Methods and any associated references are available in the online version of the paper at www.nature.com/nature.

Received 13 January; accepted 11 May 2010.

Published online 7 July 2010.

1. Yin, H. H. \& Knowlton, B. J. The role of the basal ganglia in habit formation. Nature Rev. Neurosci. 7, 464-476 (2006).

2. Hikosaka, O., Takikawa, Y. \& Kawagoe, R. Role of the basal ganglia in the control of purposive saccadic eye movements. Physiol. Rev. 80, 953-978 (2000).

3. Graybiel, A. M., Aosaki, T., Flaherty, A. W. \& Kimura, M. The basal ganglia and adaptive motor control. Science 265, 1826-1831 (1994).

4. Smith, Y., Bevan, M. D., Shink, E. \& Bolam, J. P. Microcircuitry of the direct and indirect pathways of the basal ganglia. Neuroscience 86, 353-387 (1998).

5. Albin, R. L., Young, A. B. \& Penney, J. B. The functional anatomy of basal ganglia disorders. Trends Neurosci. 12, 366-375 (1989). 
6. DeLong, M. R. Primate models of movement disorders of basal ganglia origin. Trends Neurosci. 13, 281-285 (1990).

7. Alexander, G. E. \& Crutcher, M. D. Functional architecture of basal ganglia circuits: neural substrates of parallel processing. Trends Neurosci. 13, 266-271 (1990).

8. Kawaguchi, Y., Wilson, C. J. \& Emson, P. C. Projection subtypes of rat neostriatal matrix cells revealed by intracellular injection of biocytin. J. Neurosci. 10, 3421-3438 (1990).

9. Surmeier, D. J., Mercer, J. N. \& Chan, C. S. Autonomous pacemakers in the basal ganglia: who needs excitatory synapses anyway? Curr. Opin. Neurobiol. 15, 312-318 (2005)

10. Gatev, P., Darbin, O. \& Wichmann, T. Oscillations in the basal ganglia under normal conditions and in movement disorders. Mov. Disord. 21, 1566-1577 (2006)

11. Boyden, E. S., Zhang, F., Bamberg, E., Nagel, G. \& Deisseroth, K. Millisecondtimescale, genetically targeted optical control of neural activity. Nature Neurosci. 8, 1263-1268 (2005).

12. Adamantidis, A. R., Zhang, F., Aravanis, A. M., Deisseroth, K. \& de Lecea, L. Neural substrates of awakening probed with optogenetic control of hypocretin neurons. Nature 450, 420-424 (2007).

13. Arenkiel, B. R. et al. In vivo light-induced activation of neural circuitry in transgenic mice expressing channelrhodopsin-2. Neuron 54, 205-218 (2007).

14. Huber, D. et al. Sparse optical microstimulation in barrel cortex drives learned behaviour in freely moving mice. Nature 451, 61-64 (2008).

15. Sohal, V. S., Zhang, F., Yizhar, O. \& Deisseroth, K. Parvalbumin neurons and gamma rhythms enhance cortical circuit performance. Nature 459, 698-702 (2009)

16. Cardin, J. A. et al. Driving fast-spiking cells induces gamma rhythm and controls sensory responses. Nature 459, 663-667 (2009).

17. Gong, S. et al. Targeting Cre recombinase to specific neuron populations with bacterial artificial chromosome constructs. J. Neurosci. 27, 9817-9823 (2007)

18. Ouimet, C. C., Langley-Gullion, K. C. \& Greengard, P. Quantitative immunocytochemistry of DARPP-32-expressing neurons in the rat caudatoputamen. Brain Res. 808, 8-12 (1998).

19. Tepper, J. M. \& Bolam, J. P. Functional diversity and specificity of neostriatal interneurons. Curr. Opin. Neurobiol. 14, 685-692 (2004).

20. Kreitzer, A. C. Physiology and pharmacology of striatal neurons. Annu. Rev Neurosci. 32, 127-147 (2009).

21. Kreitzer, A. C. \& Malenka, R. C. Endocannabinoid-mediated rescue of striatal LTD and motor deficits in Parkinson's disease models. Nature 445, 643-647 (2007)
22. Gradinaru, V. et al. Targeting and readout strategies for fast optical neural control in vitro and in vivo. J. Neurosci. 27, 14231-14238 (2007).

23. Gerfen, C. R. et al. D1 and D2 dopamine receptor-regulated gene expression of striatonigral and striatopallidal neurons. Science 250, 1429-1432 (1990).

24. Schwarting, R. K. \& Huston, J. P. Unilateral 6-hydroxydopamine lesions of mesostriatal dopamine neurons and their physiological sequelae. Prog. Neurobiol. 49, 215-266 (1996).

25. Bergman, H., Wichmann, T. \& DeLong, M. R. Reversal of experimental parkinsonism by lesions of the subthalamic nucleus. Science $249,1436-1438$ (1990).

26. Bergman, H., Wichmann, T., Karmon, B. \& DeLong, M. R. The primate subthalamic nucleus. II. Neuronal activity in the MPTP model of parkinsonism. J. Neurophysiol. 72, 507-520 (1994)

27. Mallet, N., Ballion, B., Le Moine, C. \& Gonon, F. Cortical inputs and GABA interneurons imbalance projection neurons in the striatum of parkinsonian rats. J. Neurosci. 26, 3875-3884 (2006)

Supplementary Information is linked to the online version of the paper at www.nature.com/nature.

Acknowledgements We thank T. Marzullo and NeuroNexus Technologies for their assistance with the custom probe design, the Nikon Imaging Center at UCSF for assistance with image acquisition, R. Javier and A. Gittis for assistance with slice electrophysiology experiments, and L. Shoenfeld for assistance with genotyping and histology. A.C.K and co-workers are funded by the W.M. Keck Foundation, the Pew Biomedical Scholars Program, the McKnight Foundation and the NIH.

Author Contributions A.V.K., P.R.L.P. and M.T.T. collected and processed tissue, and analysed immunohistochemical experiments. B.S.F. performed and analysed in vitro electrophysiology experiments. A.V.K., B.S.F. and P.R.L.P. performed and analysed in vivo electrophysiology experiments. A.V.K., P.R.L.P. and K.K. performed and analysed optogenetic/behavioural experiments. K.D. provided Cre-dependent ChR2-YFP and YFP-control constructs. A.C.K. designed and coordinated the study, and A.C.K. and A.V.K wrote the paper.

Author Information Reprints and permissions information is available at www.nature.com/reprints. The authors declare no competing financial interests Readers are welcome to comment on the online version of this article at www.nature.com/nature. Correspondence and requests for materials should be addressed to A.C.K. (akreitzer@gladstone.ucsf.edu). 


\section{METHODS}

Subjects. BAC transgenic mouse lines that express Cre recombinase under control of the dopamine D1 receptor (D1-Cre) and Dopamine D2 receptor (D2Cre) regulatory elements ${ }^{17}$ were obtained from GENSAT. Animals entered the study at $\sim 4$ weeks of age, weighing 15-20 g. All procedures were approved by the UCSF Institutional Animal Care and Use Committee.

Viral expression of DIO-ChR2-YFP and DIO-YFP. We used DIO constructs to express ChR2-YFP fusions and YFP alone in Cre-expressing neurons. The DIO constructs virtually eliminate recombination in cells that do not express Cre recombinase $\mathrm{e}^{15,16}$. The double-floxed reverse ChR2-YFP or YFP cassette was cloned into a modified version of the pAAV2-MCS vector (Stratagene) carrying the EF- $1 \alpha$ promoter and the WPRE to enhance expression. The recombinant AAV vectors were serotyped with AAV1 coat proteins and packaged by the viral vector core at the University of North Carolina. The final viral concentration was $4 \times 10^{12}$ viral particles per millilitre (by Dot Blot, UNC vector core).

Stereotaxic viral injections. Anaesthesia was induced with a mixture of ketamine and xylazine ( $100 \mathrm{mg}$ ketamine plus $5 \mathrm{mg}$ xylazine per kilogram of body weight i.p.) and maintained with isoflurane through a nose cone mounted on a stereotaxic apparatus (Kopf Instruments). For mice used in acute recording and histological analysis, the scalp was opened and a hole was drilled in the skull ( $+0.5 \mathrm{~mm} \mathrm{AP},-1.5 \mathrm{~mm}$ ML from bregma). DIO-ChR2-YFP virus $(1 \mu \mathrm{l})$ was injected into the left dorsomedial striatum ( $-3.0 \mathrm{~mm}$ DV from top of brain) through a 33-gauge steel injector cannula (PlasticsOne) using a syringe pump (World Precision Instruments) that infused the virus over 4 to $10 \mathrm{~min}$. The injection cannula was left in place for 5-10 min following the injection and then slowly removed.

Mice that were used in behavioural experiments were first implanted with guide cannulae (26-gauge, 3-mm deep, measured from top of skull; PlasticsOne) aimed at the above coordinates $(+0.5 \mathrm{~mm} \mathrm{AP,} \pm 1.5 \mathrm{~mm} \mathrm{ML}$ from bregma). After implantation of the guide cannula, viral injections (ChR2-YFP or YFP) were made through a 33-gauge injection cannula that was passed through the guide cannula, such that the tip of the injection cannula extended $0.5 \mathrm{~mm}$ from the end of the guide cannula. Injection parameters for these mice were identical to those described above. Following the injections, dummy cannulae were inserted into the guide cannulae to maintain patency, and plastic caps were attached to secure the dummy cannulae in place. All surgical procedures were performed under aseptic conditions.

To allow time for viral expression, animals were housed for at least two weeks following injection before any recording or behavioural experiments were initiated.

Striatal 6-OHDA injections. Cannulae were implanted over dorsomedial striatum and AAV1 DIO-ChR2-YFP virus was injected as described above. Nine days after viral injection, 'pre-lesion' behavioural data was collected from these animals. Animals were tethered with fibre-optic cables during the collection of all pre-lesion data. Directly following this data collection, animals were anaesthetized with ketamine and xylazine ( $100 \mathrm{mg}$ ketamine plus $5 \mathrm{mg}$ xylazine per kilogram of body weight i.p.), and 6-OHDA ( $5 \mathrm{mg} \mathrm{ml}^{-1}$; Sigma-Aldrich) was injected through the implanted cannulae ( $1 \mu \mathrm{l}$ per side). Animals were allowed to recover for five days before post-lesion behavioural testing commenced.

Implantation of electrode arrays for awake recordings. To record waveforms of fast-spiking interneurons (Supplementary Fig. 2), we implanted multielectrode arrays into striatum. Anaesthesia was induced with a mixture of ketamine and xylazine (100 mg ketamine plus $5 \mathrm{mg}$ xylazine per kilogram of body weight i.p.) and maintained with isoflurane through a nose cone mounted on a stereotaxic apparatus (Kopf Instruments). The scalp was opened and a hole was drilled in the skull ( 0.0 to $+1.0 \mathrm{~mm} \mathrm{AP},-1.0$ to $-2.0 \mathrm{~mm}$ ML from bregma). Two skull screws were implanted in the opposing hemisphere. Dental adhesive (C\&B Metabond, Parkell) was used to fix the skull screws in place and coat the surface of the skull. An array of 16 microwires $(2 \times 8$ array, $35-\mu \mathrm{m}$ tungsten wires, $100-\mu \mathrm{m}$ spacing between wires, $200-\mu \mathrm{m}$ spacing between rows; Innovative Physiology) was lowered into the striatum $(3.0 \mathrm{~mm}$ below the surface of the brain) and cemented in place with dental acrylic (Ortho-Jet, Lang Dental). After the cement dried, the scalp was sutured shut. Animals were allowed to recover for at least seven days before striatal recordings were made.

Slice recordings. Coronal sections $(300-\mu \mathrm{m}$ thick) containing dorsal striatum were prepared from brains of six-week-old heterozygous D1-Cre or D2-Cre BAC transgenic mice that were unilaterally injected at four weeks with AAV1 DIO-ChR2-YFP virus. Slices were prepared with a vibratome (Leica VT1000S) in carbogenated ACSF containing $125 \mathrm{mM} \mathrm{NaCl}, 26 \mathrm{mM} \mathrm{NaCHO}_{3}$, $2.5 \mathrm{mM} \mathrm{KCl}, 1 \mathrm{mM} \mathrm{MgCl}_{2}, 2 \mathrm{mM} \mathrm{CaCl}_{2}, 1.25 \mathrm{mM} \mathrm{NaH}_{2} \mathrm{PO}_{4}, 12.5 \mathrm{mM}$ glucose, $2 \mathrm{mM} \mathrm{Mg-ATP}$ and $0.3 \mathrm{mM} \mathrm{Na-GTP}, \mathrm{pH} 7.25,300$ mosM. After recovery for $0.5-5 \mathrm{~h}$, experiments were performed on slices perfused with ACSF, warmed to $31-33^{\circ} \mathrm{C}$.
Striatal slices were visualized using an Olympus BX51WI microscope equipped with epifluorescence. ChR2-YFP-positive neurons were selected for whole-cell recording. For voltage-clamp characterization of ChR2-mediated currents, the internal solution contained $120 \mathrm{mM} \mathrm{CsMeSO}_{3}, 15 \mathrm{mM} \mathrm{CsCl}$, $8 \mathrm{mM} \mathrm{NaCl}, 0.5 \mathrm{mM}$ EGTA, $10 \mathrm{mM}$ HEPES, $2 \mathrm{mM}$ Mg-ATP, $0.3 \mathrm{mM} \mathrm{Na-}$ GTP, $5 \mathrm{mM}$ QX-314, pH 7.3. For current-clamp characterization, the internal solution contained $130 \mathrm{mM} \mathrm{KMeSO}, 10 \mathrm{mM} \mathrm{NaCl}, 2 \mathrm{mM} \mathrm{MgCl}, 0.16 \mathrm{mM}$ $\mathrm{CaCl}_{2}, 0.5 \mathrm{mM}$ EGTA, $10 \mathrm{mM}$ HEPES, $2 \mathrm{mM}$ Mg-ATP, $0.3 \mathrm{mM}$ Na-GTP, pH 7.3. All recorded neurons exhibited electrophysiological characteristics of medium spiny neurons (Supplementary Fig. 2).

Excitation of ChR2 was achieved by epifluorescence (100-W mercury arc lamp, excitation filter; Chroma ET470/40x) and gated by a Uniblitz VS25 shutter (Vincent Associates) under through-the-lens control. Measured light intensity at the slice was approximately $1 \mathrm{~mW} \mathrm{~cm}^{-2}$. Data were collected with a MultiClamp 700B amplifier (Molecular Devices) and ITC-18 A/D board (HEKA) using IGOR PRO 6.0 software (Wavemetrics) and custom acquisition routines (mafPC, courtesy of M. A. Xu-Friedman). Current-clamp recordings were filtered at $10 \mathrm{kHz}$ and digitized at $40 \mathrm{kHz}$; voltage-clamp recordings were filtered at $2 \mathrm{kHz}$ and digitized at $10 \mathrm{kHz}$. Electrodes were made from borosilicate glass (pipette resistance, 2-4 M $\Omega$ ).

The action potential half-width was calculated as the time it took the membrane potential to surpass and return to a point halfway between the action potential threshold (defined as the voltage at which the acceleration in voltage exceeded $3 \times 10^{5} \mathrm{Vs}^{-2}$ ) and the peak. The maximum firing rate was measured as the average maximum firing rate over a 500-ms step that could be sustained without entering depolarization block. The input resistance was calculated in voltage clamp (using the K-based internal) as the deviation from the holding current caused by a $5-\mathrm{mV}$ hyperpolarizing step. The change in current was calculated as the difference between baseline holding current and the average current during the last $10 \mathrm{~ms}$ of a 50-ms hyperpolarizing voltage step. Whole-cell capacitance was calculated by integrating the area under the transient following a $5-\mathrm{mV}, 50-\mathrm{ms}$ hyperpolarizing voltage step from the holding potential $(-70 \mathrm{mV})$.

Construction of a silicon optrode. Recordings under conditions of anaesthesia were made with a custom silicon probe that was fabricated by NeuroNexus Technologies. This probe contained sixteen $413-\mu \mathrm{m}$ recording sites, arranged linearly with $50-\mu \mathrm{m}$ spacing between each site (model alx16-5mm50-413, NeuroNexus Technologies). A short (15-cm) glass optical fibre (AFS105/125Y, Thorlabs) was connectorized with an LC connector at one end and cleaved flat at the other end. This fibre was secured in place with epoxy such that the cleaved fibre tip was positioned $\sim 50 \mu \mathrm{m}$ above the first recording site (Supplementary Fig. 5). The four sites closest to the fibre were not able to record any single-unit activity, and these sites were therefore not used in further analyses.

Anaesthetized striatal optrode recordings. Twelve to sixteen days after the ChR2 injection, anaesthesia was induced with a mixture of ketamine and xylazine (100 mg ketamine plus $5 \mathrm{mg}$ xylazine per kilogram of body weight i.p.) and maintained with both isoflurane and ketamine/xylazine injections. The scalp of the animal was opened and the craniotomy that was used for the viral injection was cleaned out and expanded with a surgical drill. The optrode was then lowered through this craniotomy. We coupled the silicon optrode to a 473-nm laser (Laserglow) via a fibre-optic patch cord, and used the optrode to record striatal activity in anaesthetized D1-ChR2 and D2-ChR2 mice, as well as wild-type mice. Each optrode recording lasted $10 \mathrm{~min}$, during which time the laser alternated 60 times between $5 \mathrm{~s}$ on (constant illumination) and $5 \mathrm{~s}$ off. After each recording, the probe was lowered or moved to a new recording tract such that multiple recordings were made from each mouse. The laser power was between 1 and $2 \mathrm{~mW}$ at the tip of the optical fibre for all optrode recordings (measured with a PM100D optical power meter with an S120C sensor, Thorlabs). Recordings from wild-type mice were identical, except that the laser was not activated in these recordings. Dopamine agonist treatment during striatal optrode recordings. Animal preparation and optrode recording parameters in these experiments were identical to those described in the preceding paragraph. These recordings lasted $80 \mathrm{~min}$, during which time the laser was illuminated once per minute for $5 \mathrm{~s}$. After $20 \mathrm{~min}$ of baseline recording, a dopamine agonist (SKF81297 or quinpirole) was injected (1.0-5.0 $\mathrm{mg} \mathrm{kg}^{-1}$, i.p.). The recording then continued for an additional $60 \mathrm{~min}$. Anaesthetized SNr recordings. Twelve to sixteen days after the ChR2 injection, anaesthesia was induced with a mixture of ketamine and xylazine (100 mg ketamine plus $5 \mathrm{mg}$ xylazine per kilogram of body weight i.p.) and maintained with both isoflurane and ketamine/xylazine injections. The scalp of the animal was opened and the striatal craniotomy that was used for the viral injection was cleaned out and expanded with a surgical drill. In addition, a $\mathrm{SNr}$ craniotomy was drilled the following coordinates: -3.0 to $-4.0 \mathrm{~mm} \mathrm{AP},-1.0$ to $-2.0 \mathrm{~mm}$ ML from bregma. A $200-\mu \mathrm{m}$ fibre was coupled to the $473-\mathrm{nm}$ laser and lowered $3.0 \mathrm{~mm}$ into the striatal craniotomy. In addition, a silicon probe (model alx16-10mm100-413, 
NeuroNexus Technologies) was lowered through the SNr craniotomy. Recordings of SNr neurons were made at depths of between 4.0 and $5.0 \mathrm{~mm}$ below the surface of the brain. After electrode insertion, we tested for modulation of the local field potential (LFP) during striatal illumination. For these tests, the laser was illuminated in an alternating pattern of $1 \mathrm{~s}$ on (constant illumination) and $9 \mathrm{~s}$ off. If recording sites showed LFP modulation, we recorded single-unit activity in this region of the $\mathrm{SNr}$ in response to striatal illumination. On recordings that had a responsive unit, we recorded the response of the unit to at least 50 presentations of the above pattern. An electrolytic lesion was then made at the most ventral electrode site by passing $25 \mu \mathrm{A}$ of current through each recording array site for $10 \mathrm{~s}$. The location of this lesion was verified to be in the $\mathrm{SNr}$ through post hoc histological analysis. The laser power was between 1 and $4 \mathrm{~mW}$ at the tip of the optical fibre for all SNr recordings (measured with a PM100D optical power meter with an S120C sensor, Thorlabs).

Analysis of anaesthetized recordings. Voltage signals from each recording site on the silicon probe were band-pass-filtered, such that activity between 0.7 and $300 \mathrm{~Hz}$ was analysed as LFPs and activity between 150 and 8,000 Hz was analysed as spiking activity. Both types of data were amplified, processed and digitally captured using commercial hardware and software (Plexon). Single units were discriminated with principal component analysis (OFFLINE SORTER 3.0.1, Plexon). Two criteria were used to ensure quality of recorded units: (1) recorded units smaller than $100 \mu \mathrm{V}(\sim 3$ times the noise band) were excluded from further analysis and (2) recorded units in which more than $1 \%$ of interspike intervals were shorter than $2 \mathrm{~ms}$ were excluded from further analysis.

We tested each recorded neuron for a significant increase in firing rate during the entire period when the laser was on $(5 \mathrm{~s}$ in striatal recordings, $1 \mathrm{~s}$ in $\mathrm{SNr}$ recordings), relative to an identical time period directly preceding the laser illumination (paired $t$-tests across all 60 laser presentations). In the experiments with dopamine agonists, all light-responsive striatal neurons were tested for a response to the dopamine agonist. For these tests, the ten trials immediately preceding the agonist injection were compared with the ten trials that occurred ten minutes after the injection (unpaired $t$-tests).

Spike cross- and auto-correlation analyses were performed in NEUROEXPLORER 4.06 (Nex Technologies) with 5-ms bins over $600 \mathrm{~ms}$ (cross-correlation) or $2 \mathrm{~s}$ (auto-correlation) of spike data. For both cross- and auto-correlations, the first $100 \mathrm{~ms}$ of the correlogram was used as a baseline. For cross-correlations, a significant correlation in spiking between two neurons was defined as a peak value greater than two standard deviations above the mean of the baseline. For auto-correlations, a significant oscillation was defined as at least three peaks greater than two standard deviations above the mean of the baseline. Auto-correlations and the average delay to first spike were calculated for all lasermodulated neurons $(n=26)$, whereas cross-correlations were calculated for all simultaneously recorded pairs of laser-modulated striatal neurons $(n=21)$. We calculated the prevalence of modulated neurons, the change in firing rate of modulated neurons and the average magnitude of LFP deflection at each recording site on the optrode. We also performed a current source density analysis of the LFP data recorded on the optrode, using the CSDplotter toolbox (http:// arken.umb.no/ klaspe/iCSD.php).

Analysis of awake striatal recordings. Voltage signals from each recording site on the microwire array were band-pass-filtered, such that activity between 150 and $8,000 \mathrm{~Hz}$ was analysed as spiking activity. This data was amplified, processed and digitally captured using commercial hardware and software (Plexon). Single units were discriminated with principal component analysis (OFFLINE SORTER, Plexon). Two criteria were used to ensure quality of recorded units: (1) recorded units smaller than $100 \mu \mathrm{V}$ ( $\sim 3$ times the noise band) were excluded from further analysis and (2) recorded units in which more than $1 \%$ of interspike intervals were shorter than $2 \mathrm{~ms}$ were excluded from further analysis. Average waveforms were exported with OFFLINE SORTER. Neurons with a shouldershoulder duration of less than $500 \mu$ s were classified as putative fast-spiking interneurons, and all other neurons were classified as putative medium spiny neurons (Supplementary Fig. 2).

Optical stimulation in awake mice. Two 3-m glass fibres (AFS105/125Y, Thorlabs) were connectorized with SMA connectors at one end and cleaved flat at the other end. The cleaved ends were epoxied to a double injector guide (PlasticsOne) such that when both fibres were inserted fully into the bilateral guide cannulae, the cleaved ends of the fibre emerged $\sim 0.5 \mathrm{~mm}$ past the end of the guide cannulae. The SMA-connectorized ends were attached to SMA bulkheads on a project board that contained a splitter that divided the laser into two beams; adjustable mirrors and collimators focused each beam into its corresponding SMA bulkhead. Each beam also passed through a variable neutraldensity filter, which was adjusted such that the power was $1 \mathrm{~mW}$ at each fibre tip. The cleaved ends of the optical fibres were inserted through the guide cannulae into the dorsomedial striatum of the mice. In almost all cases ( 35 of 38 mice across all experiments), fibres were inserted without anaesthesia. In three cases, brief isoflurane anaesthesia was required to insert the fibres. In these cases, we waited at least $15 \mathrm{~min}$ after the mice regained consciousness before starting any behavioural experiments.

Behavioural analysis. After optical fibres were inserted, each mouse was placed in a round activity chamber (14-inch diameter, 8-inch high) and videomonitored from above. The positions of the nose, tail and centre of mass of each mouse were tracked using ETHOVISION 7.0 software (Noldus). A smoothing algorithm was applied to all video tracks before analysis, to reduce system noise (locally weighted scatterplot smoothing based on ten points before and after each data point, performed in ETHOVISION 7.0). Animals were recorded for a 1-10-min baseline period before any laser illumination. Then the laser ( $473 \mathrm{~nm}$, Laserglow) was illuminated in a series of ten trials. Each trial had a variable period during which the laser was on (constant illumination, 30-s average, 25-35-s range), followed by a variable period during which the laser was off (60-s average, 55-65-s range).

For each trial, we calculated all behavioural measures during three analysis periods, termed 'pre', 'laser' and 'post'. The 'pre' period was the 25-s period preceding the illumination of the laser. The 'laser' period was the period starting $5 \mathrm{~s}$ after the illumination of the laser and lasting until the end of the laser illumination (average duration, $25 \mathrm{~s}$ ). The 'post' period was the period between 5 and $30 \mathrm{~s}$ after the end of the laser illumination.

Ambulation was defined as periods when the velocity of the animal's centre point averaged more than $2 \mathrm{~cm} \mathrm{~s}^{-1}$ for at least $0.5 \mathrm{~s}$. The accuracy of this definition was validated by two observers, and it excluded other movements such as rearing and sniffing. Immobility was defined as continuous periods of time during which the average pixel change of the entire video image was less than $2 \%$ for at least $1 \mathrm{~s}$. This definition was very strict, such that any movement of the head, limbs or tail would not be scored as immobility. Fine movement was defined as any movement that was not ambulation or immobility. The frequency of grooming bouts during fine movement was scored manually. Rotations were defined as each $360^{\circ}$ rotation that contained no turn of more than $90^{\circ}$ in the opposite direction.

The frequency, average duration and total time spent performing specific behaviours were calculated using ETHOVISION 7.0. Except for the 6-OHDA experiments, all behavioural measures were normalized by dividing them by the group average 'pre' values. For the 6-OHDA experiments, all data was normalized by dividing them by the 'pre-lesion' data. We performed paired $t$-tests on the normalized data, comparing the 'laser' period to both the 'pre' period and the 'post' period for each measure. We also used paired $t$-tests to compare 'pre-lesion' measures to 'pre' measures in the 6-OHDA experiments.

Gait analysis. Gait was analysed with an automated gait analysis system (DigiGait, Mouse Specifics). This system imaged the mice from below with a high-speed camera $(\sim 150$ frames per second) while they ran on a treadmill. Software then identified specific paw steps, and from the position and timing of the paw steps calculated metrics used in this study (stride length, stride width, stance time, swing time and strides per second). The treadmill was set at $15 \mathrm{~cm} \mathrm{~s}^{-1}$ for all experiments, which is a moderate running pace. We analysed changes in gait of each mouse with the laser off versus on, with bilateral illumination (paired $t$-tests).

Histology. Animals were killed with a lethal dose of ketamine and xylazine ( $400 \mathrm{mg}$ ketamine plus $20 \mathrm{mg}$ xylazine per kilogram of body weight i.p.) and transcardially perfused with first PBS and then $4 \%$ paraformaldehyde. Following perfusion, brains were left in $4 \%$ paraformaldehyde for $16-24 \mathrm{~h}$ and then moved to a $30 \%$ sucrose solution in PBS for 2-3 days. Brains were then frozen and cut into $30-\mu \mathrm{m}$ sections (either coronal or sagittal) with a sliding microtome (Leica Microsystems, model SM2000R) equipped with a freezing stage (Physiotemp).

For immunostaining, sections were divided into ten interleaved sets, such that each set contained a series of slices through the striatum at 300- $\mu \mathrm{m}$ intervals. We prepared sections from D1-ChR2 and D2-ChR2 mice and processed sections for immunohistochemistry using antibodies against Cre recombinase (EMD Bioscience, 1:500), tyrosine hydroxylase (Pel-Freez, 1:200), DARPP-32 (Santa Cruz, 1:500), choline acetyltransferase (Millipore, 1:500), parvalbumin (Swant, 1:1,000), neuropeptide Y (ImmunoStar, 1:1,000), and c-Fos (Calbiochem, $1: 2,000)$. All antibodies were rabbit polyclonal and were labelled with a donkey anti-rabbit Alexa 568 or 647 secondary antibody (Invitrogen, 1:500). YFP fluorescence from the ChR2-YFP fusion protein remained intense after immunostaining and was visualized without any further immunohistochemistry. Immunostaining was done on free-floating sections, which were then mounted on slides and coverslipped.

To identify cannula and electrode lesion locations, sections containing tissue displacement from the cannulae or lesions were identified and set aside during slicing, stained with hematoxylin and mounted on slides. Sections were then photographed in bright field on a Nikon E600 microscope with a $\times 2$ objective. From these photographs, cannula and electrode locations were identified and marked on a coronal schematic of the striatum at $0.5 \mathrm{~mm}$ anterior to bregma. 
Microscopy. To quantify immunostaining with ChR2-YFP fluorescence, we acquired images using a BioRad Radiance 2000 confocal system mounted on an Olympus BX-60 microscope. Images were collected from single optical sections using a $\times 40$ Plan Fluor objective ( 0.75 numerical aperture). Excitation of YFP fluorescence was achieved using a 488-nm laser, and Alexa 647 fluorescence was achieved using a 647-nm laser line. Two observers performed all cell counts.

To quantify levels of $\mathrm{TH}$, one $\times 20$ field of view was captured from the dorsomedial striatum and cortex of each tested brain. All images were captured under identical conditions, and the overall fluorescence of each image was measured with IMAGEJ 1.42q (W. S. Rasband, http://rsb.info.nih.gov/ij/). In 6-OHDAlesioned mice, TH fluorescence from dorsomedial striatum was backgroundsubtracted and compared with dorsomedial TH levels in a group of control mice.

To acquire whole-brain images, mice were perfused and processed as above. Sagittal or coronal sections were mounted on slides and imaged on a Nikon D6 epifluorescence microscope with a motorized stage (Nikon Imaging Center, UCSF). Nikon ELEMENTS 3.1 software was used to control the motorized stage and stitch together a mosaic of $\times 10$ images of the entire brain slice. 